\title{
Research on the Construction Mode of Mechanical and Electrical Integration of Intelligent Control System based on Multi-agent Technique
}

\author{
Xiaofeng $\mathrm{Xu}$ \\ Weihai Vocational College, \\ Weihai ,Shandong 264210, China
}

\begin{abstract}
In this paper, we conduct research on the construction mode of mechanical and electrical integration of intelligent control system based on the multi-agent technique. The development of the modern science and technology has greatly promoted the cross of different subjects and infiltration, caused the technological transformation and revolution in the field of engineering. In the field of mechanical engineering, because of rapid development of microelectronics technology and computer technology to the mechanical industry and its mechanical and electrical integration, which is formed by the penetration of the technology of mechanical industry structure, product, organization, function and structure, mode of the production and management system, great changes have taken place in industrial production by the mechanical electrification "ushered in the development of" mechatronics "as the characteristics of the stage. Our research combines the multi-agent technique to propose the new paradigm for mechanical and electrical integration which is innovative.
\end{abstract}

Keywords- Construction Mode, Integration, Intelligent Control, Multi-agent Technique.

\section{Introduction}

Electromechanical integration includes two aspects of the mechanical and electrical integration of the technology and products electromechanical integration technology refers to the technology principle, use and electromechanical integration products and development of technology. Electromechanical integration products are including the mechanical technology, microelectronics technology, computer technology, automatic control technology, optical technology and communications and some of other high-tech products. The significance of the technique can be generally summarized as the follows. (1) Mechatronics simplifies the agency chain to make the mechanical wear, fit clearance and the stress deformation caused by error is greatly reduced, so as to achieve purely mechanical working accuracy cannot be achieved by technical means. (2) Electromechanical integration products have a variety of complex function, make multiple devices of the process to a more complete and can automatically detect and precision of workpiece, its function greatly enhanced. (3) It uses the computer technology promotes the degree of automation of the product reduce the operation button and handle, to improve the operating performance of the equipment. (4) Using microprocessor, large scale integrated circuit, power electronics devices that reduce the number of component parts in the mechanical and electrical integration products, volume small, the structure is simplified [1-3].

Mechanical and electrical integration includes two aspects of hardware and software technology. Hardware consists of body, sensor, information processing unit and the drive unit and the other parts. Software is related to each of the hardware of the carrier of the information. Body must improve the performance, quality and improve the precision of a few respects such as the 
consideration. Modern machinery products are generally based on steel material is given priority to in order to reduce the quality in addition to improve in the structure, should also consider the use of non-metallic composite material. Only with mechanical body weight, it's possible to realize the miniaturization of the drive system, and then improve the fast response characteristics in terms of basic control, reduce energy consumption and improve the general overall efficiency. With integrated circuit integrity more and more complex, the material performance is steady, electromechanical integration products to enhance reliability, at the same time due to have the security chain control, overload, and out of control, power protection function, to further improve the safety and reliability of the electromechanical integration products due to the introduction of the software technology, so as to realize the machine working procedure's modifiability, able to work with software modifications to meet the needs of the situation changes. The development of software and hardware must be coordinated. In order to reduce software development costs, improve the efficiency of the production maintenance, to gradually introduce the software standardization, including program standardization, modularization, software procedure of the curing, implementation of software engineering, etc.

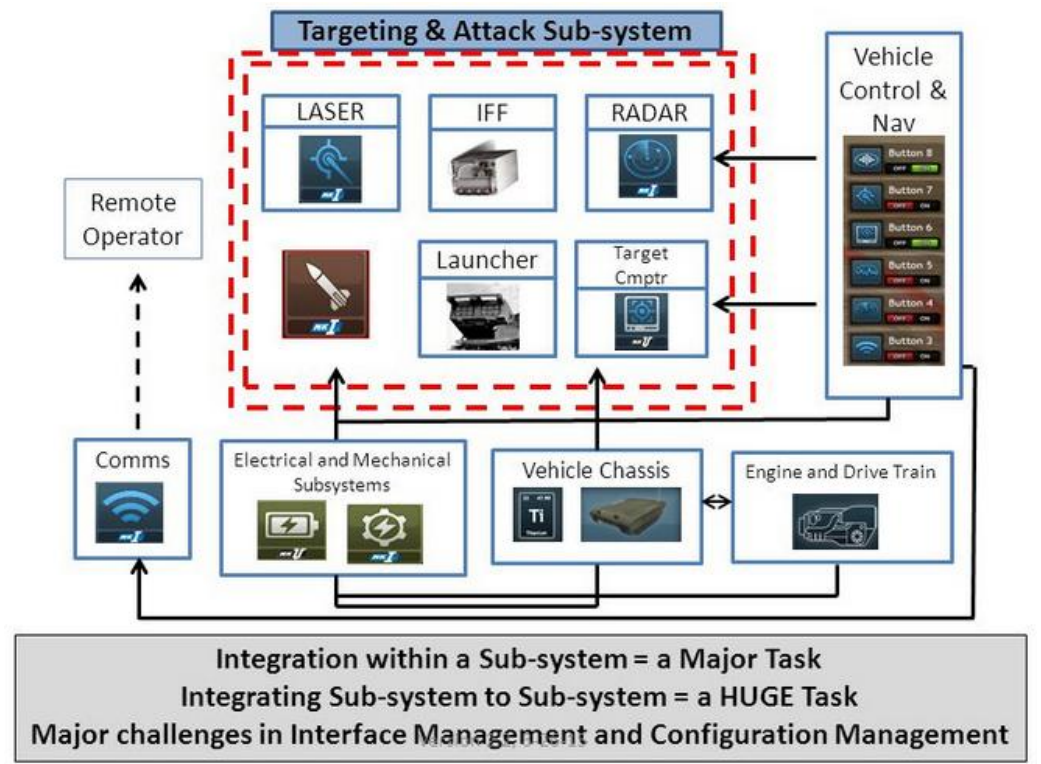

Figure 1. The Demonstration of Mechanical and Electrical Integration

As shown in the figure one, we illustrate the sample system, in this paper we conduct research on construction mode of mechanical and electrical integration of intelligent control system based on the multi-agent technique. The rapid development of the modern science and technology, promoted the crossing and infiltrating of different science, and raised almost all of the technological revolution and reform in the field of engineering. By mechanical technology and electronic technology as the main body, the multidisciplinary blend infiltration technology, combination of mechanical and electrical integration of emerging disciplines, is gradually becomes one of the focuses of the academic research nowadays. In the later parts, we will discuss the related topics in detail.

\section{Our Proposed Methodology}

The Intelligent Control. Control theory is developed gradually along with the wave of the industrial revolution. First of all, it is to realize the control of the engine, such as control of the steam engine, the internal combustion engine running schedule, this is human can't do. The 
speed regulator can make the machine run smoothly, and out of control phenomenon will not occur. By a regulator the steam machine system dynamic characteristic analysis, as well as the study of the marine driving control system, as the beginning of control theory research. With the development of electronic technology, the noise control of the amplified signal and the control of the waveform distortion to further make the feedback control theory. Intelligent control system of the three basic control methods is the artificial neural network, fuzzy logic and expert system that are introduced as follows [4-5].

- Expert system is put forward for the first time in 1983. It is on the basis of summarizing the expertise knowledge, the machine through the study, an expert to solve the problem of basic fuzziness experience, thus has the ability of experts to solve the problem can make up the defects of lack of domain experts.

- Fuzzy logic theory is put forward in 1965, broke the traditional determine the limitations of digital "right or wrong, is consistent with the fuzziness of human thinking and language logic. Fuzzy control is the main features are: it is a kind of nonlinear control method doesn't depend on mathematical model of the object that has the intrinsic parallel processing mechanism, and show the strong robustness.

- Neural network using the ideas of nonlinear mapping and parallel processing, the method of using neural network itself structure to express the implicit function coding input and output correlation knowledge with the mapping relation of input and output space is through constant learning, adjust the network structure, with the specific structure of the network finally.

In a sense, the simulation of the intelligent is to set up one or more computational intelligence framework which is run by calculation presents some intelligent behavior. Computational intelligence framework is a kind of open, hierarchical, distributed integrated computing structure, it can be for a variety of information fusion, for a variety of the intelligent technology integration, it tightly coupled connection ways rather than loose union and integration is implemented by the generalized dynamic optimization from local to the global [6].

The Multi-Agent Technique. Closely, formally Agent theory study is to discuss the concept of individual cognitive state, the Agent of the cognitive structure, analysis of the characteristics of the Agent. It involves philosophy, sociology, and cognitive science software engineering, object-oriented systems, distributed computing and artificial intelligence, etc.

Computer supported cooperative work need to establish a more equal entity interaction relations, emphasizes the system coordination of each entity in the cooperation as Agent in the communication between the behavior and characteristics and the work communication behavior of group members. Agent between peer entities are equal cooperation relations, emphasized through communication and collaboration between the Agents to complete the task. Therefore, triggered by the Agent autonomy of collaborative makes the Agent can better reflect the features of collaborative group or organization structure and contact that could be reflected from the figure two. 


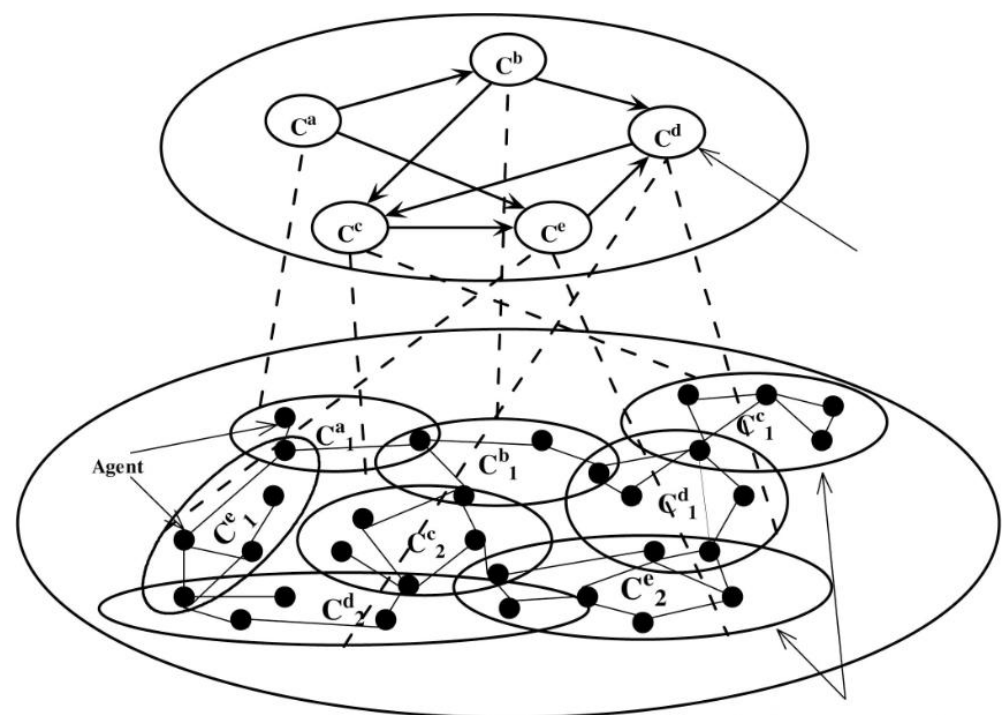

Figure 2. The Illustration of the Multi-Agent Technique

From the figure two, we could summarize the architectures of the Agent systems as the follows. (1) Deliberative architecture makes the Agent coordinate with each other help each other, in order to find the goal of the whole system. In the cooperation between the Agent negotiation or cooperation in the communication process can be referred to as before and the consultation process including through communication, or for other Agent in the system of the current mental state or intention to identify the potential interaction of reasoning, and by modifying the intentions of other Agent to avoid harmful interaction or to cooperate. (2) Agent autonomy to the communication, and the communication body responsible for meet the needs of the Agent. Communication body automatic reasoning in the field of application of artificial intelligence and database technology. (3) In a pure DPS system, the problem is broken down into tasks, and to solve these tasks, just design some special task execution system for the issue. All of the interactive strategy is integrated as a complete part of the system. This is a system of top-down design, because it is designed to meet the needs of a given by the top [7-8].

\section{The Mechanical Control Systems.} Underactuated mechanical system with less than the degree of freedom drive, it can drive less complicated task. This paper consider a movement in the vertical plane of the underactuated two pole gymnastics robot, the control goal is to make it fast smoothly from the stability of the equilibrium point vertically unstable equilibria, shake to the vertical in this balance and stability. The rapid development of the embedded chip technology cannot leave unique advantages of embedded control chip, specifically, following obvious characteristics of embedded control chips. (1) Multitasking. Embedded control chip can effectively support a variety of the tasks. Therefore, can be successful through embedded control chip instead of the low efficiency of manual meter reading work and at the same time, it also can effectively reduce the input error, significantly improve information security and the reliability. (2) Embedded control chip system has the strong personalization features. Its software and hardware system closely, as usually need according to the hardware in order to develop relevant mobile and embedded system. In addition, the need to refer to the hardware on the same brand, same series chip products to adjust and correct. (3) As a result of the embedded control chip can be cut, related research and development personnel can according to the status of the implementation details that make the embedded type system on 
the basis of meet the requirements of relevant implement streamlined configuration, so as to effectively reduce the cost.

The Future of Mechanical and Electrical Integration. The performance of the mechanical and electrical products must pass through mechanical and cooperate to achieve these two technologies connect with each other, complement each other. , it cannot be only rely on electronic or mechanical device, and want to consider mechanical device and electronic control, namely the combination of the software and hardware, the various techniques to achieve coexistence. In addition, in the design of the product at the same time to consider the product cost, reliability, accuracy, market demand and other requirements. The trend of the development can be summarized as the follows.

- Network. It is currently the era of knowledge economy, development of network technology is clueless, electromechanical integration under the promoting effect of the network also won the development opportunity.

- The bionic materialized. Electromechanical integration products in the future is the perfect combination of stability and dynamic, it is flexible in the structure, dynamic, but on the job is stable, persistent, not because of the structure of the flexible damage the stability of its work, also can't because of work to be stable and disrupt the structure of spirit, and this will hinder the other work.

- Intelligent. Intelligence is salient feature of electromechanical integration is the development direction of mechanical and electrical integration. Let the machine has complete the wisdom of such a fan does have some difficulties, but let the machine owner of intelligence is entirely the achievable.

- Environmental protection. The development of industry brings people rich material life, but also cause environmental burden, and so people are racing to calls for the birth of the green products and to use mechanical and electrical integration is no exception. Green mechanical and electrical integration of the product as small as possible harm the environment refers to the use which can be recycled after use.

Mechanical and electrical integration technology is the basic process of constant development and improvement. Product precision and the production efficiency of mechatronics system constantly improved control algorithm of servo drive performance and the development requirements, and the excellent performance of servo drive is to expand function of the mechanical system, the simplified the traditional institutions, and requirements of mechanical system have reasonable inertia and better system dynamic performance. Machine, electricity and close cross have the mechanical and electrical integration system of information of each part connection and influence each other to become the important content of design must be considered. Early electromechanical integration design mainly focuses on the composition and structure of system, along with the design practice and design theory of mature, the mechanical and electrical interface technology as the core of the electromechanical integration design has caught the attention of more and more experts and scholars.

\section{Conclusion}

In this paper, we conduct research on the construction mode of mechanical and electrical integration of the intelligent control system based on the multi-agent technique. Electromechanical integration technology of each technology research has been very deep and increasingly mature, at the same time people also realize the technology development and research the composition does not guarantee of the mechatronics system optimization. Therefore, 
somebody puts forward to the theoretical study of mechatronics system design is advanced the theory of mechatronics system integration and fusion. We think theory of system design and system integration and fusion of theory research is necessary. Mechanical and electrical functions solving process from function to effect, finally to the embodiment of the original understanding and structured process. At the system level of principle solution, the so-called unit function solution interface features actually contains a function, effect and principle of the interface features. In the future, we will integrate the mathematical optimization idea into the core part of the agent system for further modification.

\section{References}

[1] Lee, Sunwoo, et al. "Graphene metallization of high-stress silicon nitride resonators for electrical integration." Nano letters 13.9 (2013): 4275-4279.

[2] Wei, D. U. A. N. "Explore the analysis of the development trend of China's mechanical and electrical integration." Hunan Agricultural Machinery 3 (2012): 068.

[3] Schkoda, Ryan F., and Charles Fox. "Integration of mechanical and electrical hardware for testing full scale wind turbine nacelles." Power Systems Conference (PSC), 2014 Clemson University. IEEE, 2014.

[4] Guoliang, Zhong. "Analysis of Fault Diagnosis Methods for Mechanical and Electrical Integration Equipment." Friend of Science Amateurs 2 (2012): 033.

[5] XIE, Jia, et al. "Study of compact and efficient mechanical-electrical integration ocean energy generation system." Electronic Design Engineering 13 (2015): 051.

[6] Myers, Joshua C., et al. "Integration of piezoelectric energy harvesting and antenna elements on a common substrate." Electronic Components and Technology Conference (ECTC), 2013 IEEE 63rd. IEEE, 2013.

[7] Zhao, Shuai, et al. "Improving dispersion and integration of single - walled carbon nanotubes in epoxy composites by using a reactive noncovalent dispersant." Journal of Polymer Science Part A: Polymer Chemistry 50.21 (2012): 4548-4556.

[8] Desmaris, V., et al. "Carbon Nanofibers (CNF) for enhanced solder-based nano-scale integration and on-chip interconnect solutions." Electronic Components and Technology Conference (ECTC), 2014 IEEE 64th. IEEE, 2014. 